\title{
Analysis of TEM tomography artifacts with experiments on model specimens
}

\author{
Jonathan Winterstein ${ }^{1}$, Joshua Schumacher ${ }^{1}$ and J. Alexander Liddle ${ }^{1}$ \\ 1. NIST Center for Nanoscale Science and Technology, Gaithersburg, MD 20899
}

TEM tomography is becoming a critical technique for the characterization of materials. Despite the rapid development of software and imaging methods for obtaining tomograms from a wide range of materials [1], important questions remain about the resolution in three dimensions and the uncertainties in quantitative measurements. Recently, Mezerji et al. reported a useful method for measuring the anisotropic resolution in the reconstructed volume using curve fitting to edge profiles, and derived values different from those predicted by the well-known Crowther criterion [2]. Perhaps more importantly, it is necessary to quantify the accuracy of dimensional measurements (e.g., particle volumes) which are in turn determined by the accuracy of segmentation or edge-finding methods and not directly related to resolution (the ability to distinguish two closely spaced objects).

We will report measurements of 3D feature sizes and resolution in reconstructed volumes using several different reconstruction algorithms from different model samples: $\mathrm{MgO}$ smoke cubes, $\mathrm{Au}$ nanoparticles and FIB-prepared pillars. In the case of $\mathrm{MgO}$ smoke cubes and the FIB pillars it is possible to know the $3 \mathrm{D}$ sample shape a priori and therefore quantitatively to compare the accuracy of reconstruction and analysis methods. Unlike reconstructions with digital phantoms, these experiments also permit analysis of the effects of noise, beam damage and tilt-series alignment on the 3D reconstruction.

Figure 1 shows the results of a reconstruction of a tilt series of ADF-STEM images of an MgO cube using a model-based reconstruction method [3]. The cubic shape is preserved in three dimensions and the reconstruction shows good fidelity. Some artifacts appear, possibly due to the effect of diffraction contrast in the STEM images or the missing wedge. A SIRT reconstruction (not shown) of the same dataset suffers from significantly worse artifacts due to the missing wedge. This particle presents a particularly difficult challenge for tomographic reconstruction due to the sharp, flat facets oriented perpendicular to the optical axis, and is thus an excellent test case.

Volume measurements of $\mathrm{Au}$ nanoparticles also show significant differences between different reconstruction methods and thresholding procedures for individual particles and groups of particles. Measurement of the anisotropic resolution is straightforward with $\mathrm{Au}$ nanoparticles using the curve fitting method of [2] and is compared between different reconstructions in figure 2.

Using samples with a priori known dimensions allows us to compare the fidelity of different segmentation methods as well. Comparisons of standard segmentation procedures will be presented and the possibility of fully automated, objective segmentation and quantification discussed. We will also discuss possible superior alternative model samples for characterization of TEM tomography reconstruction and analysis.

\section{References:}

[1] PA Midgley, M Weyland, Ultramicroscopy 96 (2003), p. 413.

[2] H Heidari Mezerji, W Van den Broek, S Bals, Ultramicroscopy 111 (2011), p. 330. 
[3] SV Venkatakrishnan, LF Drummy, MA Jackson, M De Graef, J Simmons, CA Bouman, IEEE Transactions on Image Processing 22 (2013), p. 4532.
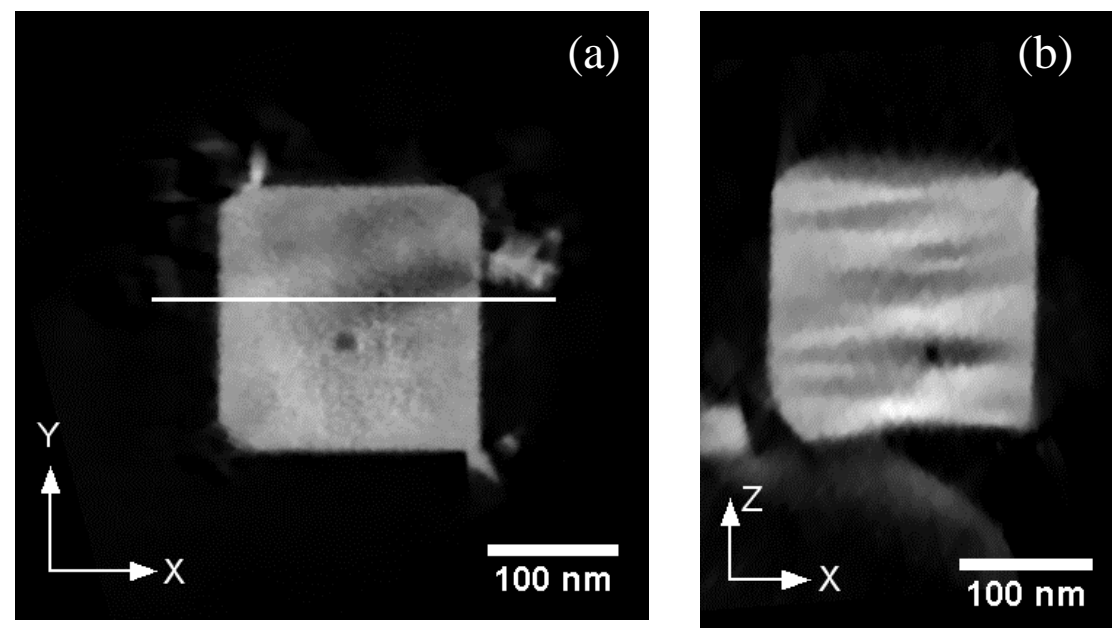

Figure 1. Tomographic reconstruction of a $\mathrm{MgO}$ smoke cube using the MBIR algorithm from [3]: a single slice in the X-Y plane (a), and in the X-Z plane (b) where the horizontal line in (a) indicates the position of the slice shown in (b), and a 3D perspective view of the particle after segmentation (c).
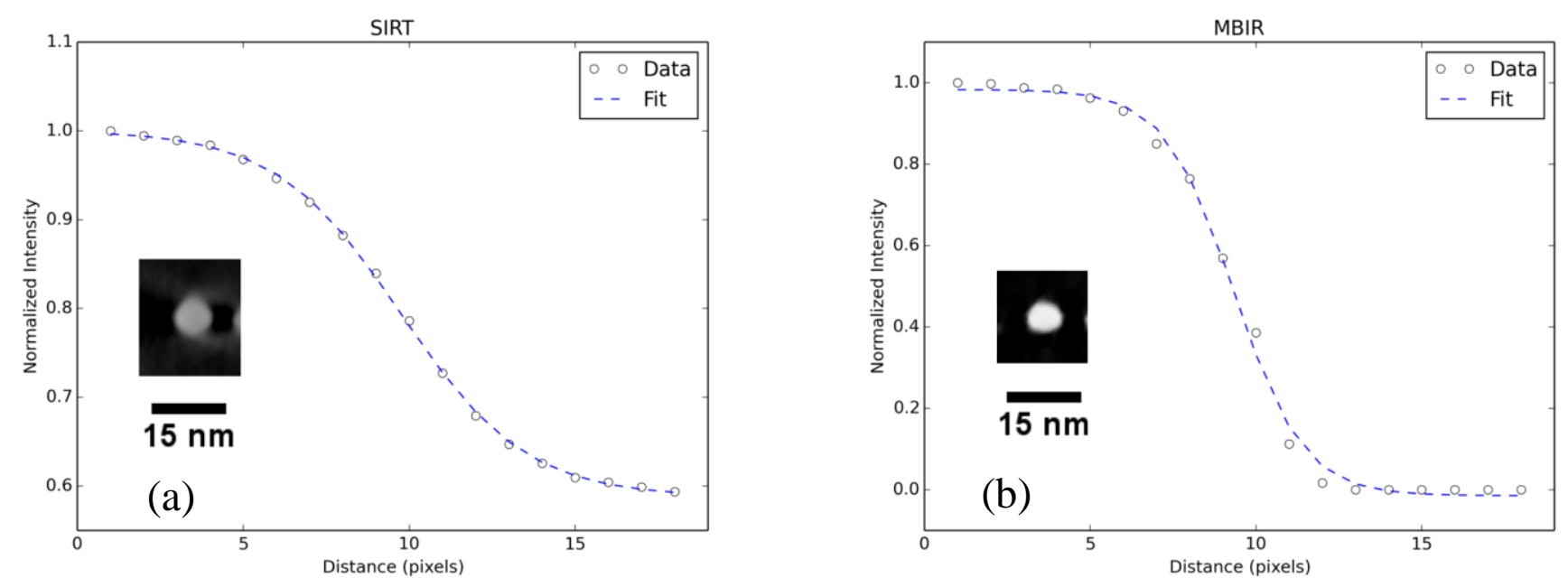

Figure 2: Results of curve fitting to edge intensity profiles along the $\mathrm{z}$ direction (optical axis) from tomographic reconstructions of the same Au particle using two different reconstruction methods. 141 ADFSTEM images acquired at $1^{\circ}$ tilt intervals from $-70^{\circ}$ to $+70^{\circ}$ were used for the reconstruction. 